EXTENDED REPORT

\title{
Influence of radiographic phenotype on risk of hip osteoarthritis within families
}

\author{
P Lanyon, K Muir, S Doherty, M Doherty
}

See end of article for authors' affiliations ......................

Correspondence to: Dr P Lanyon, Department of Rheumatology, Queens Medical Centre

Nottingham, NG7 2UH, UK; peter.lanyon@ nottingham.ac.uk

Accepted 12 May 2003
Objective: To determine whether the magnitude of the genetic influence on the development of hip osteoarthritis (OA) varies according to the radiographic phenotype within families.

Participants and methods: 331 families in which at least one sibling (index participant) had undergone total hip replacement for OA and whose preoperative $x$ ray findings were available; 505 siblings of these index participants, who have high exposure to genetic risk of hip OA; and 1718 participants who had previously undergone intravenous urography, representative of the average general population exposure to genetic risk. Prevalence of hip OA was determined by individual radiographic features and minimum hip joint space. OA phenotype was partitioned according to pattern of femoral head migration and osteophyte bone response. Age adjusted odds ratios for hip OA in siblings, stratified according to phenotypic pattern in their index sibling, were assessed by unconditional logistic regression.

Results: The superior pattern of femoral head migration was more common in men, and the axial pattern more common in women. A poor bone response (absent osteophytosis) was associated with an indeterminate pattern of migration. The age adjusted odds ratios for definite hip OA were twofold higher in siblings of index participants who had no osteophyte response than in siblings whose index case had any degree of osteophyte (OR 2.05, $95 \% \mathrm{Cl} 1.12$ to 3.76). The risk of the siblings from these families having undergone hip replacement themselves was threefold higher. Patterns of migration and bone response were not concordant within families, even among same sex siblings.

Conclusion: Careful phenotypic characterisation is essential for genetic studies of hip OA. The results of these studies are likely to be influenced by the phenotypic pattern of hip disease, particularly osteophyte bone response.

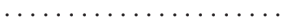

The study participants comprised a subgroup selected from a larger cohort of 392 families, within which at least one sibling had hip OA of sufficient severity to warrant total hip replacement (THR). The design and recruitment of this larger cohort has been described in detail elsewhere ${ }^{2}$; in brief, three groups of subjects were recruited: reported a strong genetic influence on the development of radiographic hip OA. ${ }^{12}$ However, there is a large phenotypic variation within primary hip OA, and the relationship between genetic susceptibility and hip OA phenotype is not known.

Hip OA can be phenotyped according to the radiographic pattern of femoral head migration within the acetabulum. Although several descriptive classifications exist, the most widely used has three basic patterns: superior, axial (along the axis of the femoral neck), and medial, ${ }^{3-5}$ (fig 1). Phenotypic classification can also be made according to the bone response, either atrophic, characterised by bone attrition and minimal new bone or osteophyte response, or hypertrophic, characterised by florid new bone formation. ${ }^{6}$

Radiographic classification has clinical relevance. Superior migration is more common in men and more likely to be unilateral and to progress in hospital series. ${ }^{78}$ Medial migration is more common in women, is associated with Heberden's nodes, is more likely to occur bilaterally, and may be associated with an increased failure of the acetabular cup after joint replacement. ${ }^{9-11}$ These phenotypic patterns may reflect different aetiologies. ${ }^{710}$

This study aimed at determining whether the magnitude of the genetic influence on the development of hip OA within families varies according to the radiographic phenotype within families, and whether phenotypic patterns "breed true" within families.

\section{SUBJECTS AND METHODS}

\section{Study subjects}

Approval for this study was obtained from the local research ethics committee.

1. Index subjects with primary hip OA, who had been listed for hip replacement surgery between 1990 and 1996.

2. Their siblings, identified by questionnaire, and included if willing to attend for pelvic radiography (or had preexisting radiographs). Siblings were selected for this current study if the preoperative pelvic radiograph of their index case could be obtained, in order to compare the risk and pattern of hip OA in siblings with the pattern in the index case.

3. Subjects who had previously undergone intravenous urography (IVU), assumed to represent the average genetic susceptibility of the general population.

About three times more IVU subjects than siblings, of the same sex and age at $x$ ray examination (to within 2 years), were recruited. The risks of hip OA were compared in these two groups.

\section{Radiographic assessment}

To enable direct comparison between siblings and IVU subjects, siblings underwent modified pelvic radiography with the same technique used for an IVU "control" film. ${ }^{2}$ Individual radiographic features of OA were graded 0-3

Abbreviations: IVU, intravenous urography; MJS, minimum joint score; OA, osteoarthritis; THR, total hip replacement 


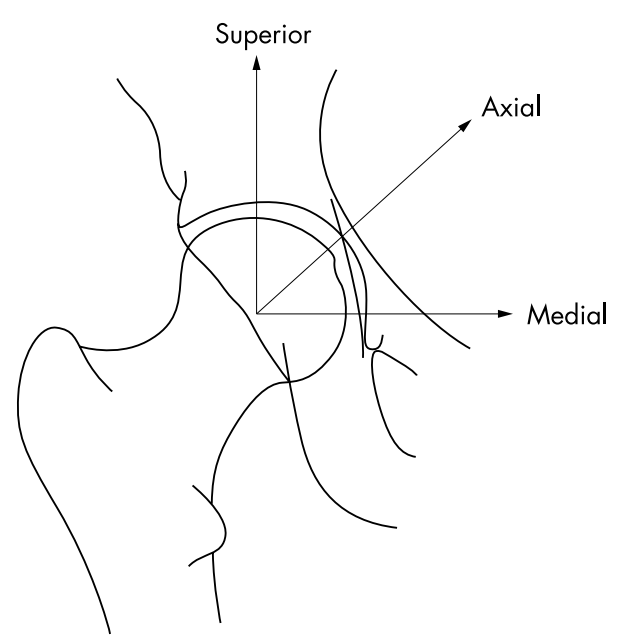

Figure 1 Patterns of femoral head migration.

according to a standard atlas. ${ }^{5}$ Minimum hip joint space (MJS) at the site of maximum narrowing was measured by metered dial caliper (RS Components, Switzerland) to within $0.1 \mathrm{~mm}$. Hip OA was defined by MJS according to recommended methods; either $\leqslant 2.5 \mathrm{~mm}$ ("probable" hip $\mathrm{OA}$ ) or $\leqslant 1.5 \mathrm{~mm}$ ("definite" hip OA). ${ }^{12}$ Both definitions were assumed to have been satisfied if a THR was present.

The pattern of femoral head migration was determined from the earliest abnormal preoperative film (fig l) according to four possible patterns: superior, axial, medial, or indeterminate (implying no identifiable pattern, as a result of either complete concentric narrowing or distortion/collapse of the femoral head).

Bilateral hip OA was designated if there was a bilateral THR or a unilateral THR and the contralateral hip showed grade 1 or higher narrowing. To determine the pattern of bone response, index subjects were categorised according to the maximum single osteophyte grade in either hip. The osteophyte response was subsequently dichotomised into either absent (grade 0) or present (grades 1-3).

Reproducibility was assessed in a subset of 40 radiographs (80 hips) chosen to include a range of feature severity, read blind, about one month apart. The $95 \%$ limits of agreement for joint space width were $\pm 0.5 \mathrm{~mm} .{ }^{13} \kappa$ Values $^{14}$ for ordinal variable were all $>0.6$, apart from 0.3 for acetabular osteophyte.

Age adjusted relative risks for hip OA in siblings (compared with IVU subjects), stratified according to the phenotypic pattern in the index case (site of maximum joint space narrowing, osteophyte response), were inferred from odds ratios calculated by unconditional logistic regression using SPSS (SPSS Inc, Chicago, Illinois). Unless stated otherwise, we performed unmatched analyses, selecting only one hip for each subject, that which showed the greatest severity of the feature under study - that is, the worst hip.

\section{RESULTS}

The pelvic radiographs of 336 (86\%) of the original 392 index subjects with a hip replacement were obtained; five of these did not contain a preoperative radiograph. The radiographs of the remaining 56 subjects could not be obtained (missing from $x$ ray file despite repeated searches). The study participants therefore comprised 331 index subjects with a hip replacement (202 female, 129 male) and their 505 siblings (298 female and 215 male, mean age at $x$ ray examination 65 years).
Table 1 Site of maximum joint space narrowing in index subjects with THR

\begin{tabular}{lll}
\hline Site of narrowing & $\begin{array}{l}\text { Women } \\
(\mathbf{n = 2 0 2 )}\end{array}$ & $\begin{array}{l}\text { Men } \\
(\mathbf{n}=129)\end{array}$ \\
\hline Indeterminate & $30(15)$ & $17(13)$ \\
Superior & $77(38)$ & $90(70)$ \\
Axial & $77(38)$ & $19(15)$ \\
Medial & $18(9)$ & $3(2)$ \\
\hline \multirow{2}{*}{ Results are shown as No (\%). }
\end{tabular}

The IVU subjects comprised 981 women and 737 men of mean age 65 years. The overall prevalence of hip OA in these subjects varied, according to hip OA definition, from $3.8 \%$ to $11.1 \%$ in women and from $2.1 \%$ to $5.9 \%$ in men.

\section{Index participants}

One hundred and ninety one index participants had bilateral hip OA, 21 of whom had discordance in the pattern of femoral head migration between the left and right hip, and the pattern was therefore assigned from the hip that showed the most radiographic change. Superior femoral head migration was more common in men than women, and axial migration more common in women than men (table 1). In an equal proportion of men and women (14\%), no pattern ("indeterminate") could be identified. Table 2 shows the prevalence of bilateral OA within each migration pattern. The axial pattern was more frequently bilateral $(77 \%)$ than the superior pattern $(50 \%)$. Within each migration pattern, the prevalence of bilateral disease was similar in each sex.

The association between the maximum single osteophyte grade at any site in the hip and the pattern of migration is shown in table 3 . Fifty one (15\%) of these index participants had no osteophyte response (grade 0), and this was seen most frequently with an indeterminate pattern of migration (40\%, compared with $10 \%-19 \%$ in other patterns).

\section{Siblings}

The overall risk of hip OA among the whole sibling group compared with IVU subjects was increased five- to eightfold, varying according to sex and disease definition. ${ }^{2}$ Table 4 shows subgroup comparisons of the risk of hip OA according to whether their index hip replacement sibling had no osteophyte response (grade 0 ) or whether this was present (grades 1-3). Among the 82 siblings ( 50 women, 32 men) of the 51 index cases who had no osteophyte response, although there was no increased risk of "probable" hip OA, there was a twofold higher risk of "definite" hip OA compared with 431 siblings whose index case had at least grade 1 osteophyte. The risk of these siblings having required a hip replacement was threefold higher.

The risk of hip OA in siblings compared with IVU subjects did not vary according to the pattern of femoral head migration in the index case (table 5).

Table 2 Prevalence of bilateral $O A$ in index cases according to site of narrowing

\begin{tabular}{llll}
\hline \multicolumn{4}{c}{ Bilateral OA } \\
\cline { 2 - 4 } Site of narrowing & $\begin{array}{l}\text { Women } \\
(\mathbf{n}=115)\end{array}$ & $\begin{array}{l}\text { Men } \\
(\mathbf{n}=76)\end{array}$ & $\begin{array}{l}\text { Both sexes } \\
(\mathbf{n}=191)\end{array}$ \\
\hline Indeterminate & $13(42)$ & $8(50)$ & $21(45)$ \\
Superior & $47(51)$ & $36(49)$ & $83(50)$ \\
Axial & $46(81)$ & $28(72)$ & $74(77)$ \\
Medial & $9(64)$ & $4(57)$ & $13(62)$ \\
\hline \multicolumn{2}{l}{ Results are shown as No (\%) in each site of narrowing. } \\
\hline
\end{tabular}




\begin{tabular}{|c|c|c|c|c|c|}
\hline \multirow[b]{2}{*}{ Osteophyte grade } & \multicolumn{4}{|c|}{ Site of maximum narrowing, № (\%) } & \multirow[b]{2}{*}{ Total } \\
\hline & Indeterminate & Superior & Axial & Medial & \\
\hline 0 & $19(40)$ & $16(10)$ & $12(13)$ & $4(19)$ & $51(15)$ \\
\hline 1 & $8(17)$ & $44(26)$ & $26(27)$ & $5(24)$ & $83(25)$ \\
\hline 2 & $16(34)$ & $74(44)$ & $46(48)$ & $7(33)$ & $143(43)$ \\
\hline 3 & 4 (9) & $33(20)$ & $12(13)$ & $5(24)$ & $54(16)$ \\
\hline Total & 47 & 167 & 96 & 21 & 331 \\
\hline
\end{tabular}

Table 6 shows the association between the pattern of migration in index cases and their affected siblings (MJS $\leqslant 2.5 \mathrm{~mm}$ ). Siblings and index cases who could not be assigned a pattern (bilateral THR or unilateral THR with normal contralateral hip) were excluded from this analysis. No association between the index pattern and sibling pattern was seen-that is, patterns do not appear to "breed true" within families. For example, $26 \%$ of siblings of superior index cases also have a superior pattern; this is similar to the frequency of superior pattern in siblings of axial index cases. Clearly, the association between sex and pattern may have confounded this analysis. However, subgroup analysis according to the sex of either the sibling or index case also failed to demonstrate any association between sibling and index patterns, even within same sex siblings.

Table 7 shows the relationship between bone response in index cases and their affected siblings (MJS $\leqslant 2.5 \mathrm{~mm}$ ). No association was seen, with a similar proportion $(75 \%)$ of affected siblings having a greater than grade 1 osteophyte response, regardless of the osteophyte status of their index case. We did not find evidence that this response always "breeds true" within 18 individual multicase families that comprised at least three affected siblings. In nine of these families all affected siblings displayed a hypertrophic response, in three families all affected siblings displayed an atrophic response, and in six families there was a mixed pattern of bone responses.

\section{DISCUSSION}

As far as we know this is the first study to attempt to explore the relationship between hip OA risk and hip OA phenotype within families. In accordance with other studies, we found a difference in the pattern of femoral head migration between the sexes, with a superior pattern more common in men and an axial pattern more common in women. This may reflect the same aetiological process modified by anatomical differences in pelvic anatomy between men and women. Alternatively, the aetiology may be genuinely different in men and women, with possibly a greater aetiological component from occupational heavy lifting in men, modifying the location of joint space narrowing. No significant differences in femoral head migration patterns were seen between siblings and IVU subjects, which implies that these siblings have "typical" hip OA, phenotypically representative of disease in the community.

There are several caveats to the interpretation of the influence of phenotypic pattern on risk in siblings. Although this study had adequate power to detect an increased risk in

Table 4 Relative risk ( $95 \% \mathrm{Cl}$ ) of hip OA amongst 82 siblings whose index case had no osteophytosis compared with 431 siblings of index cases with at least grade 1 osteophyte

\begin{tabular}{llll}
\hline Definition & Women & Men & Both sexes \\
\hline MJS $\leqslant 2.5 \mathrm{~mm}$ & $1.11(0.56$ to 2.20$)$ & $1.25(0.54$ to 2.88$)$ & $1.16(0.69$ to 1.97$)$ \\
MJS $\leqslant 1.5 \mathrm{~mm}$ & $1.99(0.87$ to 4.52$)$ & $2.12(0.85$ to 5.24$)$ & $2.05(1.12 \text { to } 3.76)^{*}$ \\
THR & $2.63(1.06$ to 6.59$) \dagger$ & $3.44(1.19$ to 9.96$) \ddagger$ & $3.05(1.53$ to 6.07$) \S$ \\
\hline${ }^{*} p=0.019 ; \dagger p=0.037 ; \neq p=0.022, \S p=0.001$. & & \\
\hline
\end{tabular}

Table 5 Relative risk of hip OA in siblings according to site of maximum joint space narrowing in their index sibling

\begin{tabular}{lllll}
\hline & \multicolumn{2}{l}{ Site of maximum narrowing } & & \\
\cline { 2 - 5 } & Indeterminate & Superior & Axial & Medial \\
\hline Women & & & & \\
MS $\leqslant 2.5 \mathrm{~mm}$ & $4.02(1.95$ to 8.27$)$ & $4.67(3.08$ to 7.08$)$ & $4.35(2.63$ to 7.20$)$ & $2.94(1.04$ to 8.29$)$ \\
$M J S \leqslant 1.5 \mathrm{~mm}$ & $6.51(2.72$ to 15.6$)$ & $3.36(1.80$ to 6.27$)$ & $5.81(3.07$ to 10.9$)$ & $5.29(1.61$ to 17.4$)$ \\
THR & $17.3(6.27$ to 47.8$)$ & $8.37(3.75$ to 18.6$)$ & $6.41(2.47$ to 16.6$)$ & $3.29(0.39$ to 27.3$)$ \\
Men & & & & \\
MJS $\leqslant 2.5 \mathrm{~mm}$ & $4.50(1.56$ to 13.0$)$ & $5.74(3.35$ to 9.83$)$ & $7.42(3.87$ to 14.1$)$ & $7.99(2.13$ to 29.9$)$ \\
MSS $\leqslant 1.5 \mathrm{~mm}$ & $6.99(1.83$ to 26.5$)$ & $10.2(4.94$ to 21.0$)$ & $12.7(5.55$ to 29.0$)$ & $8.14(1.51$ to 43.6$)$ \\
THR & $11.9(2.23$ to 63.4$)$ & $8.37(3.75$ to 18.6$)$ & $6.41(2.47$ to 16.6$)$ & $23.8(4.06$ to 140$)$ \\
& & & & \\
Both sexes & & & & \\
MJS $\leqslant 2.5 \mathrm{~mm}$ & $4.18(2.31$ to 7.56$)$ & $4.84(3.50$ to 6.70$)$ & $5.29(3.56$ to 7.87$)$ & $4.20(1.85$ to 9.49$)$ \\
MJS $\leqslant 1.5 \mathrm{~mm}$ & $6.71(3.24$ to 13.8$)$ & $5.28(3.34$ to 8.35$)$ & $7.75(4.70$ to 12.7$)$ & $6.14(2.32$ to 16.2$)$ \\
THR & $15.6(6.66$ to 36.8$)$ & $9.78(5.19$ to 18.4$)$ & $9.60(4.68$ to 19.6$)$ & $8.13(2.21$ to 29.9$)$ \\
\hline
\end{tabular}


Table 6 Relationship between site of maximum narrowing in index cases and their affected siblings ( $\leqslant 2.5 \mathrm{~mm}$ )

\begin{tabular}{llccc}
\hline & \multicolumn{4}{l}{ Site of maximum narrowing in index case, No $(\%)$ within index case } \\
\cline { 2 - 5 } Site in sibling & Indeterminate & Superior & Axial & Medial \\
\hline Indeterminate & $5(42)$ & $2(3)$ & $3(7)$ & $1(14)$ \\
Superior & $7(58)$ & $19(26)$ & $12(28)$ & $2(29)$ \\
Axial & 12 & $48(66)$ & $28(65)$ & $3(43)$ \\
Medial & $4(5)$ & & $1(14)$ \\
Total & 12 & 73 & 43 & 7 \\
\hline
\end{tabular}

\begin{tabular}{|c|c|c|}
\hline \multicolumn{3}{|c|}{ Maximum osteophyte grade } \\
\hline \multirow[b]{2}{*}{ Siblings } & \multicolumn{2}{|c|}{ Index cases, No (\%) within index cases) } \\
\hline & 0 & $1-3$ \\
\hline $\begin{array}{l}0 \\
1-3 \\
\text { Total }\end{array}$ & $\begin{array}{l}5(26) \\
14(74) \\
19\end{array}$ & $\begin{array}{l}32(27) \\
87(73) \\
119\end{array}$ \\
\hline
\end{tabular}

siblings compared with IVU participants, it was not designed to have the necessary power to detect differences between phenotypic subgroups. Additionally, the likely association between pattern of femoral head migration, sex, and nodal status might also have confounded these analyses. However, we documented the presence of self reported Heberden's nodes using a validated diagram, but did not find a strong association between nodal status and risk of hip OA in siblings. ${ }^{15}$

Despite these limitations, our data suggest that the genetic influence on the development of hip OA may vary according to phenotypic subgroups. The risk of "definite" and severe (that is, having required a hip replacement) is significantly greater in siblings whose index case had an atrophic pattern of hip OA, with no osteophyte response, compared with those siblings whose index case had any degree of osteophyte response. We did not, however, detect this association for risk of "probable" hip OA. This suggests that genetic factors involved in bone response and architecture are important in the development and severity of hip OA. In view of the association between osteophyte and bone mineral density, ${ }^{16}$ one possible explanation is that once hip OA has developed, subjects with poor bone response, or osteoporotic bone, are more likely to have rapidly progressive, severe disease. This hypothesis is supported by the fact that the siblings of these index cases have a threefold higher risk of having undergone hip replacement themselves than siblings from families with any degree of bone response. One implication of this observation is that genetic databases that have ascertained affected hip OA sibling pairs on the basis of both having undergone total joint replacement ${ }^{17}$ may be biased towards particular phenotypic subtypes of hip OA, and thus may not reflect the heterogeneity both in phenotype and severity that comprises the "community burden" of hip disease.

We were unable to detect an influence of pattern of migration on risk, probably because of inadequate power. Moreover, patterns of migration do not appear to "breed true" even within same sex siblings-that is, although hip OA clusters within families, different patterns of femoral head migration occur within families. This also seems to be the situation for the pattern of bone response. However, this particular analysis might have been biased by the single cross sectional time frame of this study-that is, osteophyte formation may not always be a contemporaneous phenomenon with joint space narrowing. Additionally, no pattern of bone response could be assigned to siblings whose $x$ ray examinations showed the presence of bilateral hip replacements, and there was a higher prevalence of hip replacement among the siblings of atrophic index cases.

This observation that different phenotypes are present within families is important, because it implies that differences in phenotypic expression may reflect the same disease, modified by different gene/environment interactions.

\section{CONCLUSION}

This study has explored the association between expression of radiographic phenotype and genetic risk. The main finding is that within families where one sibling has undergone hip replacement for OA, the risk of hip OA among their siblings is significantly increased if the index case had no osteophyte response. There is therefore likely to be a subset of families with hip OA (about 15\% in this study) in whom poor bone response adversely modifies the OA process. These results have implications for current studies of the genetics of hip $\mathrm{OA}$, with careful phenotypic description being essential in all cases. The results of these studies are likely to be influenced by the phenotypic pattern of hip disease, particularly osteophyte bone response. Further study is needed of the interplay between cartilage loss and osteophyte response, and the candidate genes and environmental interactions that influence these processes.

\section{ACKNOWLEDGEMENTS}

We are grateful to the Arthritis Research Campaign who funded this study (grant No L0510).

\section{Authors' affiliations}

P Lanyon, Department of Rheumatology, Queens Medical Centre, Nottingham, NG7 2UH, UK

K Muir, Department of Epidemiology and Public Health Medicine, Queens Medical Centre, Nottingham, NG7 2UH, UK

S Doherty, M Doherty, Academic Rheumatology, City Hospital, Nottingham NG5 1PB, UK

\section{REFERENCES}

1 MacGregor A, Antoniades L, Matson M, Andrew T, Spector T. The genetic contribution to radiographic $O A$ in women: results of a classic twin study. Arthritis Rheum 2000;43:2410-16.

2 Lanyon P, Muir K, Doherty S, Doherty M. Assessment of a genetic contribution to osteoarthritis of the hip: sibling study. BMJ 2000;3231:1179-83.

3 Resnick D. Patterns of migration of the femoral head in osteoarthritis of the hip. Am J Roentgenol Radium Ther Nucl Med 1975; 124:62-74.

4 Altman RD, Alarcón G, Appelrouth D, Bloch D, Borenstein D, Brandt K, et al. The American College of Rheumatology criteria for the classification and reporting of osteoarthritis of the hip. Arthritis Rheum 1991;34:505-14.

5 Altman RD, Hochberg MC, Murphy WA, Murphy WA. Atlas of individual radiographic features in osteoarthritis. Osteoarthritis Cartilage 1995;3:3-70.

6 Solomon L, Schnitzler C, Browett J. Osteoarthritis of the hip: the patient behind the disease. Ann Rheum Dis 1982;41:118-25. 
7 Ledingham J, Dawson S, Preston B, Milligan G, Doherty M. Radiographic patterns and associations of osteoarthritis of the hip. Ann Rheum Dis 1992;51:1111-16.

8 Dougados M, Gueguen A, Nguyen M, Berdah L, Lequesne M, Mazieres B, et al. Radiological progression of hip osteoarthritis: definition, risk factors and correlations with clinical status. Ann Rheum Dis 1996;55:356-62.

9 Gofton JP. Studies in osteoarthritis of the hip. Can Med Assoc J 1971;104:679-83.

10 Gofton JP. A classification of osteoarthritis of the hip and its relevance to pathogenesis. J Rheumatol 1983;10:65-6.

11 Bissacotti J, Ritter M, Faris P, Keating EM, Cates HE. A new radiographic evaluation of primary osteoarthritis. Orthopedics 1994; 17:927-30.
12 Croft P, Cooper C, Wickham C, Coggon D. Defining osteoarthritis of the hip for epidemiologic studies. Am J Epidemiol 1990;132:514-22.

13 Bland J, Altman DG. Statistical methods for assessing agreement between two methods of clinical assessment. Lancet 1986;i:307-10.

14 Cohen J. A coefficient of agreement for nominal scales. Educational and Psychological Measurement 1960;20:37-46.

15 Lanyon P. The genetic contribution to the aetiology of hip osteoarthritis. University of Nottingham, 1999. (DM Thesis.)

16 Antoniades L, MacGregor A, Matson M, Spector T. A cotwin control study of the relationship between hip osteoarthritis and bone mineral density. Arthritis Rheum 2000;43:1450-5.

17 Chitnavis J, Sinsheimer J, Clipsham K, Loughlin J, Sykes B, Burge PD, et al. Genetic influences in end-stage osteoarthritis. J Bone Joint Surg $\mathrm{Br}$ 1997;79:660-4.

\section{$\mathrm{ECHO}$}

\section{Jobs determine primary osteoarthritis}

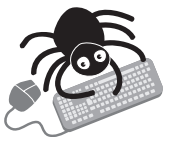

Please visit the Annals of the Rheumatic Diseases website [www. annrheumdis. com] for a link to the full text of this article. vidence from the largest national study ever undertaken confirms growing suspicions - about occupational influence on osteoarthritis (OA) and the need for more precise L understanding in formulating preventive measures. Occupation-not merely old agedetermines primary $\mathrm{OA}$, claim the researchers.

Among almost 10500 (10 412) patients, the prevalence of OA was highest in women aged 60-69 and men aged 70-79. Excess prevalence of OA was related to time in an occupation as well as occupational group. Other findings were that housekeeping was linked with excess OA for women over 60 (observed: expected $(\mathrm{O} / \mathrm{E})$ ratio 4.4) and truck driving for men aged over 70 (O/E ratio 6.7). Overall, the odds of OA for women were 1.0 for white collar workers, 2.9 "mixed," and 2.6 for blue collar workers and for men 1.0, 1.2, and 1.7, respectively. Severity of OA was evident in those 2000 or so patients who were actively employed: $22 \%$ were off work owing to total work disability and $44 \%$ had partial work disability. The lowest proportion disabled by OA were white collar workers and the highest were blue collar workers.

Data came from the 1998 French National Survey on Health Impairment and Disability, a cross sectional pharmacoepidemiological study. The survey's coverage was representative of the national population, permitting comparisons of prevalence of OA cases with expected prevalence in the general population for age, sex, and occupation. A unique network of rheumatologists in France made it possible to study such a large patient sample.

\ Occupational and Environmental Medicine 2003;60:882-886. 